\title{
MANEJO DE FRACTURA BLOW-OUT SECUNDARIA A HERIDA POR ARMA DE FUEGO MEDIANTE EL USO DE SONDA DE FOLEY: REPORTE DE CASO
}

\author{
${ }^{1}$ Carlos Ernesto Rueda Torres, ${ }^{2}$ Julio Martín Ruiz Roa \\ ${ }^{1}$ Odontólogo U. Santo Tomás, Cirujano Maxilofacial U. Javeriana, Docente U. Santo Tomás. \\ ${ }^{2}$ Estudiante X semestre F. de Odontología U. Santo Tomás.
}

Autor responsable de correspondencia: Julio Martín Ruiz Roa

Correo electrónico: martinrr69@hotmail.com

\section{RESUMEN}

Las fracturas orbitarias son traumas comúnmente asociados con golpes contundentes de alta energía sobre el globo ocular, se compromete el piso de la órbita y la pared medial en mayor prevalencia. Las necesidades de tratamiento se toman de acuerdo con el grado de compromiso ocular. A continuación se presenta el caso de un paciente de sexo masculino de 22 años de edad, perteneciente a sanidad militar, víctima de una herida por arma de fuego de alta velocidad perforante, que comprometió estructuras esqueléticas faciales y tejidos blandos que incluyeron la apófisis ascendente del maxilar superior, el piso y la pared lateral de la órbita, el complejo cigomáticomalar, el seno maxilar y la apófisis coronoides. El procedimiento quirúrgico incluyo lavado y desbridamiento, reducción y estabilización de las fracturas, reconstrucción facial y orbitaria. El objetivo de este artículo es dar a conocer la utilidad de la sonda de Foley como estabilizador de las fracturas orbito malares, durante la reconstrucción fracturas Blow-out. [Rueda CE, Ruiz JM. Manejo de fractura Blow-out secundaria a herida por arma de fuego mediante el uso de sonda de Foley: Reporte de caso. Ustasalud 2010; 9: 50 - 56]

Palabras clave: Fracturas Blow-out, Diplopía, Sonda de Foley, Piso de órbita.

\section{MANAGEMENT OF BLOW-OUT FRACTURE INJURY SECONDARY}

\section{A FIREARM BY USING A FOLEY CATHETER: A CASE REPORT}

\begin{abstract}
Orbital fractures are associated to high-energy blunt hits on the eyeball; they involve the orbital floor and lateral wall at least in all the cases. Treatment will be taken accord to the eyeball compromise. This paper shows a patient case, 22 years old, who worked at the police department. He was fire gun injured penetrating and high-speed type. There were compromised the upper ascendant maxillary apophysis, orbital floor and lateral wall of the orbit, zigomatic-malar complex bone, maxillary sinus. The surgery procedure included the wound cleaning, the stabilization and the reduction of the fracture, orbital and facial reconstruction. The objective of this case report was to increase the knowledge of Foley catheter likeness antral stopper during orbital-malar fractures during blow-out fractures reconstruction.
\end{abstract}

Key words: Blow-out fractures, Diplopy, Foley catheter, Orbital floor.

Recibido para publicación: febrero 10 de 2010. Aprobado para publicación: 22 de junio de 2010.

\section{INTRODUCCIÓN}

Las fracturas del tercio medio facial pertenecen a una entidad común de la región maxilofacial que involucra huesos propios de la nariz, órbita, maxilar superior y complejo cigomático-malar. ${ }^{1}$ Olate y colaboradores describieron las estructuras óseas que constituyen el tercio medio facial en tres grupos: la primera corresponde al complejo "cigomático-orbitario (CZO)", la segunda al complejo "nasoorbito-etmoidal (NOE)" y la tercera a la "estructura orbitaria" propiamente dicha. El arco superciliar y la región del hueso frontal corresponden a la región facial superior. ${ }^{2}$

Las fracturas aisladas de órbita comprenden entre el $4 \%$ al $16 \%$ de todas las fracturas faciales. Sin embargo, si se tienen en cuenta las fracturas asociadas con otros huesos, la incidencia se encuentra entre el $30 \%$ y el $55 \% .^{3}$

Las causas más frecuentes de la fractura de órbita en el adulto son traumatismos de regular o mayor intensidad ocasionados por objetos contundentes 
durante lesiones personales, accidentes de tránsito, caídas accidentales y heridas por arma de fuego clasificadas como "traumas de alta energía primarias por estallido". 4,5 En los niños, las causas más frecuentes son las caídas y los golpes contundentes con objetos. ${ }^{6}$

\section{Las fracturas de órbita se clasifican en:}

- Fracturas circulares Blow-in: hay un desalojo de los fragmentos de la fractura hacia la cavidad orbitaria, con la subsiguiente disminución del volumen orbitario.?

- Fracturas Blow-out: se presenta compresión del músculo recto inferior con la disminución de la movilidad ocular, se compromete cualquier pared de la órbita (superior, inferior, interna, externa).

- Fracturas lineales simples: igualmente, puede producirse rotación de fragmentos óseos, clasificada como "Trap Door", además de hernia de la grasa periorbitaria en dirección al seno maxilar. ${ }^{8,9}$

Mac Kenzie fue el primero en describir la fractura de piso de órbita de tipo Blow-out. Por otro lado, Smith y Reegan, en 1954, definieron la fractura Blow out como "el atrapamiento del musculo recto inferior en la fractura por estallido". Lang, la clasificó como una entidad clínica, mientras que, Converse, la definió como "un aumento mecánico y violento de la presión intraorbitaria, que termina fracturando el piso orbitario (la pared más delgada de la cavidad orbitaría), produce una hernia de los tejidos orbitarios dentro del seno maxilar". ${ }^{10}$

El esqueleto facial posee dos cavidades óseas que comprenden las órbitas, con bases anterolaterales y vértices posteromediales. Medialmente se encuentran separadas por los senos etmoidales y por los huesos propios de la nariz. La cavidad orbitaria se compone de cuatro paredes. La pared superior o techo de la órbita, conformada por el fragmento orbitario del hueso frontal y por el ala menor del esfenoides. La pared interna o medial, conformada por el hueso etmoides, una parte del hueso frontal, lagrimal y esfenoidal; junto con la pared inferior, son huesos de consistencia delgada y frágil, siendo los más susceptibles a la fractura Blow-out. La pared lateral o externa, está constituida por el ala mayor del esfenoides y por la apófisis frontal del hueso cigomático; esta pared es de mayor espesor y de mayor resistencia a los traumas directos. La pared inferior o piso de la órbita está conformada por el hueso maxilar superior, cigomático y palatino, es el techo del seno maxilar. La característica delgada y frágil de este hueso lo hace vulnerable a las fracturas Blow-out, al igual que la pared medial. ${ }^{11}$
El globo ocular se encuentra rodeado por los músculos extrínsecos que le permiten la movilidad en ciertas direcciones, éstos se dividen en seis: músculo recto superior, músculo recto inferior, músculo recto externo, músculo recto interno, músculo oblicuo superior y músculo oblicuo inferior. ${ }^{12}$ Se encuentran inervados por el nervio oculomotor (recto superior, recto interno, recto inferior, oblicuo inferior), nervio troclear (oblicuo superior) y nervio abducens (recto externo). ${ }^{13}$

La pared interna y la del piso de la órbita son las que se ven principalmente afectadas durante un golpe directo a la órbita ya que se crea una fuerza que se esparce a través del globo ocular que produce la fractura por consistencia anatómica. ${ }^{14} \mathrm{La}$ explicación de este suceso ha sido de interés para los oftalmólogos, otorrinolaringólogos y cirujanos maxilofaciales. El mecanismo de la lesión está constituido por dos teorías: la hidráulica "aumento de la presión intraorbitaria que ocasiona un aplastamiento directo de cualquier hueso de la órbita cuando el globo ocular es impulsado posteriormente"; y la teoría de la palanca, en la que la fuerza es transmitida a los huesos de la órbita por una deformidad transitoria del reborde orbitario. ${ }^{15}$

Existen diferentes sistemas de clasificación para las fracturas de tercio medio facial. Para un adecuado manejo de las mismas se debe conocer cada una de ellas. Según Knigth y North, las fracturas orbitomalares $(\mathrm{OM})$ se definen como un paralelismo entre el tratamiento y el pronóstico en torno a la anatomía de la fractura:

Clase I: ausencia de desplazamiento significativo.

Clase II: fracturas de arco cigomático.

Clase III: fracturas del cuerpo no rotadas.

Clase IV: fracturas del cuerpo rotadas medialmente.

Clase V: Fracturas del cuerpo rotadas externamente.

Clase VI: Fracturas complejas del cuerpo. ${ }^{4}$

Por otro lado, Manson y colaboradores (1990) las clasifican, de acuerdo con el desplazamiento reflejado en la Tomografia Axial Computarizada, en:

1. Fracturas de baja energía: desplazamiento mínimo o sin desplazamiento.

2. Fracturas de media energía: desplazamiento leve a moderado, con un nivel variable de conminución.

3. Fracturas de alta energía: desplazamiento severo, existe grave conminución. ${ }^{4}$

De acuerdo con Leipziger y manson, la clasificación para las fracturas naso-orbito-etmoidales (NOE) se basa en tres clases que pueden ser ser unilaterales o bilaterales: 
Clase I: fracturas con un segmento único central, se preserva la inserción del ligamento cantal medial.

Clase II: fracturas con porción única o conminución externa a la inserción del ligamento cantal medial.

Clase III: conminucion del fragmento central que expone la inserción del ligamento cantal medial, incluye una línea de fractura que transcurre inmediatamente debajo de ésta, muy a menudo se encuentra adherida a varias porciones óseas muy pequeñas. ${ }^{4}$

Los cambios clínicos a causa de la fractura Blow-out son variados. Es indispensable la valoración multidisciplinaria entre oftalmología y cirugía maxilofacial para un correcto manejo del trauma. ${ }^{8}$ Zambrano y Leyva, describen la evaluación del globo ocular en cinco aspectos: movimientos oculares, segmento anterior, segmento posterior, pupilas y agudeza visual. ${ }^{14}$

En las fracturas de piso de órbita de tipo Blow-out, se encuentran signos clínicos como: diplopía (indica un atrapamiento del musculo recto inferior), enoftalmos, equimosis palpebral, enfisema subcutáneo, exoftalmus, epistaxis, y dilatación pupilar, entre otros. $^{7,16}$ Casarramona y colaboradores señalaron 59 casos de fracturas de piso de órbita de tipo Blow out, donde se encontraron que los signos más comunes eran equimosis periorbitaria, diplopía, parestesia del nervio infraorbitario y enfisema infraorbitario. ${ }^{6}$

Inicialmente, para manejar este tipo de fracturas se debe disponer de métodos diagnósticos como la proyección de Waters y la Tomografia Computarizada Tridimensional que permite visualizar de forma clara la estructura esquelética y los tejidos blandos adyacentes a la órbita. ${ }^{8}$

La corrección de las fracturas Blow-out está determinada por las características clínicas existentes en el paciente. Los enoftalmos de $2 \mathrm{~mm}$ a $3 \mathrm{~mm}$, diplopía evidente y hallazgo radiográfico de atrapamiento del musculo recto inferior son determinantes en la decisión de tratamiento. Sin embargo, se debe tener un tiempo pertinente de espera (7-10 días) para evaluar si los signos clínicos mencionados disminuyen, de lo contrario se procede a la intervención quirúrgica. ${ }^{8}$

El tratamiento quirúrgico de las fracturas de piso de órbita (Blow-out), depende de la evolución, de las dimensiones y de la orientación del desplazamiento óseo, características importantes para decidir la mejor técnica de reducción de la fractura y su reconstrucción con sistemas de osteosíntesis. ${ }^{1,17}$

Frederick Foley (1934), diseñó la sonda que lleva su mismo nombre, inicialmente para ser usada como catéter urinario, hoy día es usada también por ciru- janos maxilofaciales para la reducción de fracturas faciales y en la reconstrucción del piso de órbita. ${ }^{18}$ La sonda de Foley es un catéter hecho a base de silicona, conformado por un canal principal, un extremo compuesto por dos orificios de entrada y el otro extremo compuesto por un balón que es inflado por medio de agua destilada o suero fisiológico una vez este situado dentro del seno maxilar. ${ }^{19} \mathrm{El}$ dispositivo es posicionado a través del meato inferior de la cavidad nasal en el seno maxilar del lado afectado, allí es insuflado con suero fisiológico para proporcionar un soporte temporal para los tejidos periorbitarios y para el sistema que reemplazara el hueso perdido. ${ }^{18-20}$

La prevención de las deformidades cráneo-orbitarias postraumáticas, desplazamientos del globo ocular y alteraciones de la movilidad ocular requieren la exacta reconstrucción tridimensional del piso orbitario y de la estructura frontal. Se han establecido las características para la correcta reconstrucción orbitaria: la precisa reconstrucción de la proyección y de la convexidad de la curvatura del arco supraorbitario son esenciales para la reconstrucción orbito-facial; el apoyo del hueso frontal determina la orientación y la amplitud del tercio medio facial, mientras que la posición de la glabela determina la proyección del complejo naso-etmoidal. Adicionalmente, la convexidad del techo de la órbita en los planos laterales y antero-posteriores deben ser reproducidos para prevenir el desplazamiento del globo ocular y el daño de la movilidad ocular. ${ }^{21}$

El presente artículo reporta el caso de un paciente de sexo masculino de 22 años de edad, herido por arma de fuego de alta velocidad perforante, pertenecia a sanidad militar. Hubo compromiso de las estructuras esqueléticas faciales y de los tejidos blandos. El procedimiento quirúrgico incluyó lavado y desbridamiento, reducción de las fracturas, reconstrucción facial y estabilización del piso de la órbita mediante el uso de una sonda de Foley.

\section{Reporte de caso}

Paciente masculino de 22 años de edad remitido a la Clínica de la Policía Seccional Sanidad Santander (Colombia) para valoración y manejo de trauma ocular y estallido orbitario secundario a herida por arma de fuego sufrido en actos del servicio. El paciente no presentó antecedentes médicos relevantes. El examen facial evidenció orificio de entrada del proyectil por arco superciliar interno y parpado superior izquierdo que describió un trayecto oblicuo, atravezó de izquierda a derecha la estructura esquelética correspondiente a la apófisis ascendente del maxilar superior, huesos propios nasales, piso y pared lateral de la órbita derecha, complejo cigomá- 
tico-malar, seno maxilar, y apófisis coronoides con orificio de salida a través de la región maseterina derecha. Fue valorado por los servicios de oftalmología y cirugía maxilofacial, las imágenes diagnósticas (tomografía computarizada con reconstrucción tridimensional de cara y órbitas) reportaron fractura conminuta del complejo cigomático-malar derecho con desplazamiento de la pared lateral y piso de órbita derecha, sin compromiso del vértice del ápex orbitario. Impactación de la apófisis ascendente del maxilar superior y herniación de la grasa periorbitaria derecha en el seno maxilar, fractura de la apófisis coronoides desplazada medialmente (Figura 1).

Su diagnóstico fue:

Trauma ocular secundario a herida por arma de fuego (HPAF).

Fractura orbito-malar izquierda clase VI de Knigth y North.

Avulsión de tejido blando facial secundario a HPAF.

El paciente fue intervenido por oftalmología, se realizó sutura de esclera superior en el limbo superior del ojo izquierdo; al mejorar las condiciones locales y el edema periorbitario, se encontró leve discoria en el ojo izquierdo y epifora en el ojo derecho. En el examen clínico se observó aumento del volumen en la hemicara derecha, con orificio de salida de proyectil en tejidos blandos región maseterina, depresión y ausencia de margen infraorbitaria, crepitación en la región perinasal y parestesia infraorbitaria derecha. Movimientos oculares conservados con reflejos fotomotor y consensual $(+)$.

Siete días después del trauma, el paciente fue intervenido por el servicio de cirugía maxilofacial, previa cobertura antibiótica con cefalosporina de primera generación y clindamicina endovenosa. El procedimiento quirúrgico incluyó abordaje subciliar con extensión de Lynch en órbita derecha, abordaje intraoral vestibular, exposición de los focos de fractura, lavado y desbridamiento, remoción de los focos óseos conminutos necróticos, mantenimiento al máximo de la cobertura muscular y del periostio del resto del mayor tramo de fractura conminuta posible, reducción y fijación de la pared lateral de la órbita, cuerpo malar, apófisis ascendente del maxilar superior y huesos propios nasales mediante el sistema matrix (Synthes ${ }^{\circledR}$ ). Por vía nasal y a través del meato inferior se realizó antrostomía y se instaló la sonda de Foley \# 14 (Figura 2), manteniendo visión directa intraoral hasta ubicarla en el área correspondiente al piso orbitario, insuflándola con el volumen de solución salina que permitió elevar el domo superior del balón hasta el nivel de la solución de continuidad del piso orbitario el cual fue verificado por el abordaje subciliar hasta obtener la recuperación hacia el contenido orbitario del tejido graso herniado en el seno maxilar (Figura 3).

Se seleccionó la micromalla de piso de órbita, se moldeó y ajustó al tamaño orbitario; se fijó a la estructura medial y lateral de la órbita (Figura 4), que fue reducida y fijada anteriormente. Se terminó la reconstrucción maxilo-malar a través del empleo de micromallas de titanio en la pared anterior del seno maxilar (Figura 5).

Se verificó el contorno y la proyección malar respecto al lado opuesto y se procedió a remover la sonda de Foley por vía nasal. La cirugía terminó sin complicaciones, se cerraron los abordajes quirúrgicos por planos como corresponde a las heridas por arma de fuego en región facial.

En este caso se considero la remoción de la sonda de Foley con el fin de evitar el desarrollo de un proceso infeccioso en el seno maxilar debido a que la estabilidad de las fracturas se logró con el material de fijación; la manipulación de la sonda fue suficiente para lograr el soporte de los fragmentos, la elevación del globo ocular y la remoción de los coágulos del seno maxilar.

El control ambulatorio al cabo de tres semanas evidenció una agudeza visual OD-OI 20-200, discoria del ojo izquierdo, ortoforia y resolución del edema, el TAC tridimensional mostró consolidación de las fracturas reducidas (Figura 6). Actualmente continúa manejo ambulatorio por oftalmología y cirugía maxilofacial.

\section{DISCUSIÓN}

Las fracturas orbitarias de tipo Blow-out son ocasionadas por un objeto contundente de mayor tamaño que impacta la órbita, producen un aumento de la presión intraorbitaria que ocasiona la fractura de uno de los huesos que la componen. ${ }^{22}$

En el tratamiento de las fracturas del complejo cigomático-malar, la exploración del piso de la órbita es un procedimiento que es frecuente aunque no siempre está indicado, puede ser abordado a través de incisiones en la piel o directamente desde el seno maxilar. Estos abordajes combinados, en los que se puede tener acceso directo a la órbita y acceso a través del seno maxilar han sido publicados por varios autores. ${ }^{23,24}$

Cuando el piso de la órbita se expone y el tejido periorbitario se reposiciona, un balón es colocado bajo visión directa para ser usado como soporte hasta que termine su cicatrización. Sin embargo, se debe prevenir el llenado excesivo del balón para que no produzca un aumento excesivo de la presión sobre 


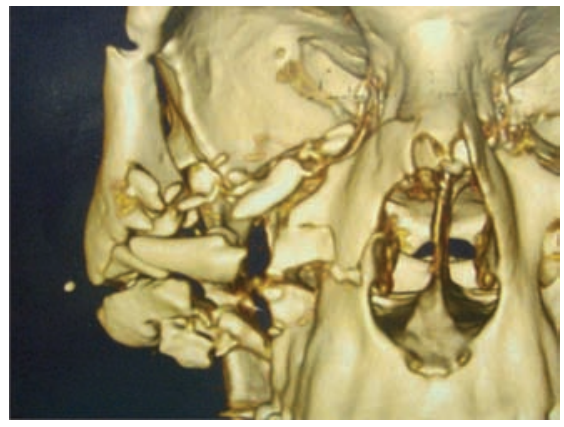

Figura 1. Tomografia tridimensional orbito-facial.
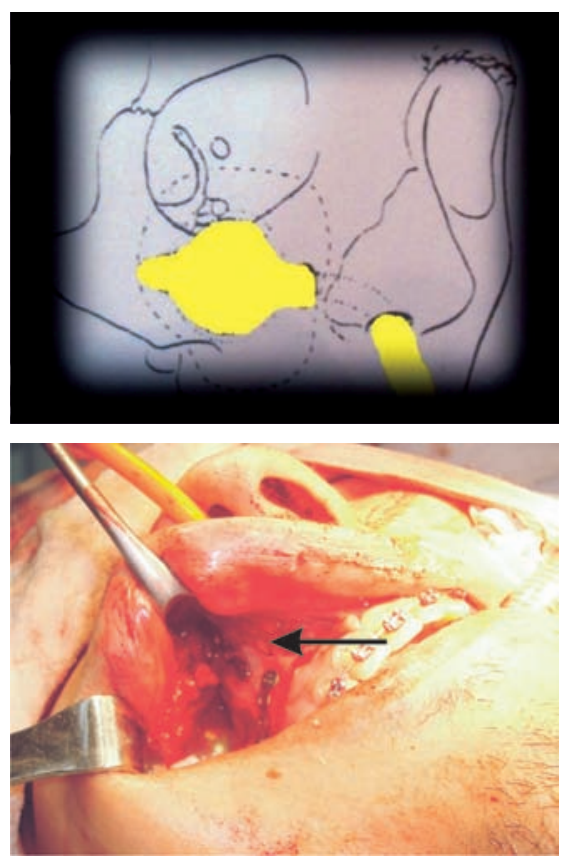

Figura 2. Colocación de la sonda de Foley vía meato inferior.
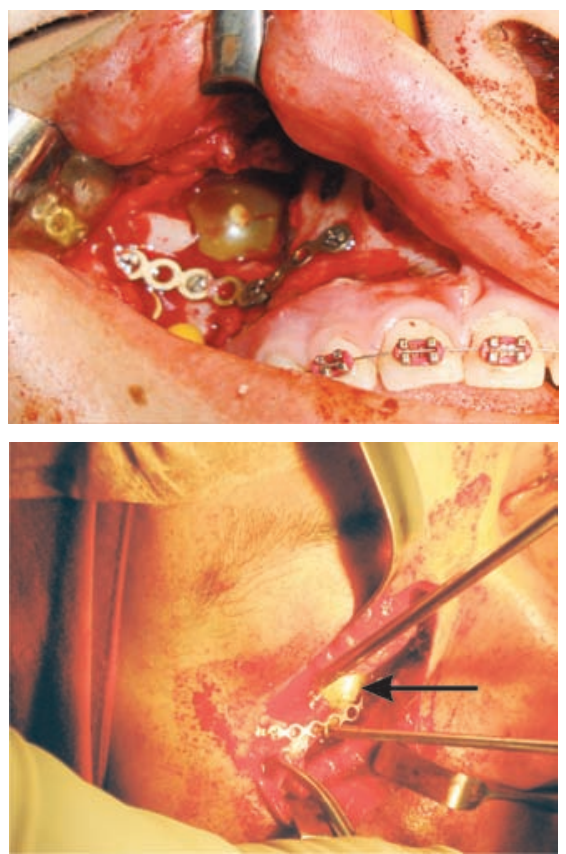

Figura 3. Sonda de Foley en el seno maxilar, se obtiene la recuperación hacia el contenido órbitario del tejido graso herniado.
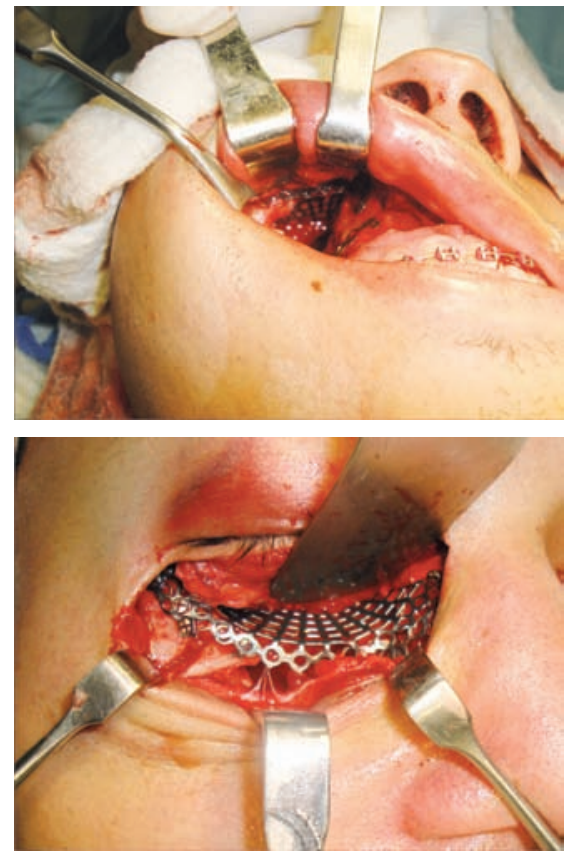

Figura 4. Micromalla de piso de órbita fijada a la estructura orbitaria.

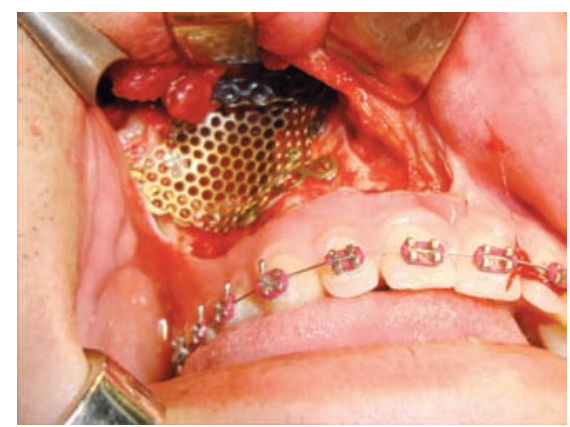

Figura 5. Micromalla de titanio en la pared anterior del seno maxilar.
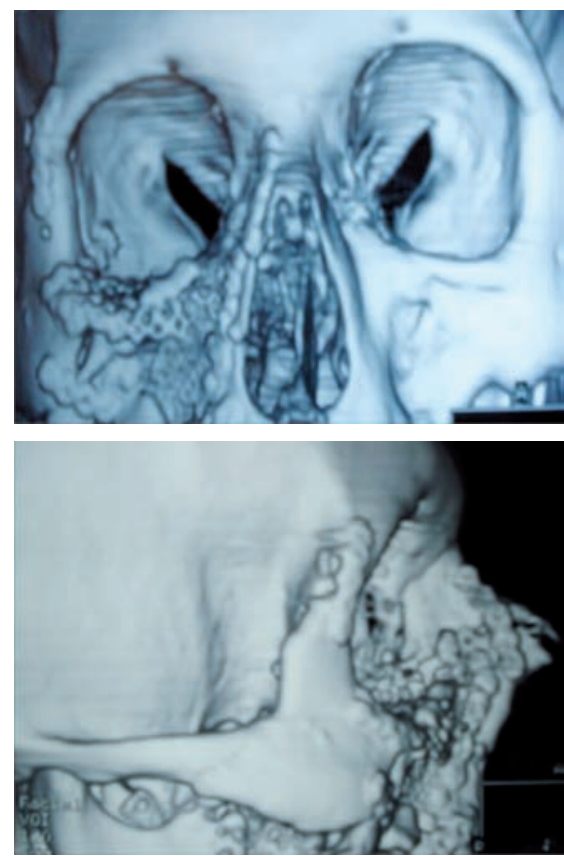

Figura 6. Tomografía tridimensional, tres semanas después de la intervención quirúrgica. 
el contenido orbitario. Para obtener la cicatrización ósea es necesario que exista suficiente tejido óseo de lo contrario se requerirá de un implante o un trasplante. La literatura reporta que el balón que provee el soporte al piso orbitario debe permanecer por dos semanas hasta que termine la cicatrización ósea aunque se ha sugerido uso de balones sinusales y de implantes de piso de órbita incrementan el riesgo de infección. ${ }^{25}$

Cuando se presenta un gran defecto y la periórbita ha sido interrumpida es necesario realizar la reconstrucción del piso orbitario para prevenir el enoftalmo y la ptosis palpebral. Varios materiales se han empleado en la reconstrucción del piso orbitario dentro los cuales están: hueso alogénico, hueso autógeno, cartílago autógeno, metil-metacrilato, polímeros de silicona, mallas de teflón y mallas metálicas, entre otros. ${ }^{25}$ Aunque los injertos autógenos son los materiales preferidos en principio debe tenerse en cuenta que éstos incrementan la morbilidad y el tiempo quirúrgico. Por tal motivo, se ha desarrollado y aceptado que los sustitutos aloplásticos pueden ser usados en la reconstrucción del piso orbitario, aun cuando existan complicaciones como la infección, la extrusión y el desplazamiento del implante; ${ }^{21}$ la elección del implante usualmente depende de la habilidad y preferencia del cirujano, por lo tanto una técnica cuidadosa puede brindar buenos resultados.

Rowe y Williams enumeraron tres situaciones en las cuales debe considerarse el uso de material intrasinusal para las fracturas de complejo cigomáticoorbitario:

Cuando el complejo cigomático continua inestable después de la reducción.

Cuando hay gran conminución del complejo cigomático.

Cuando hay conminución del piso orbitario sin pérdida ósea. ${ }^{25}$

A través de la historia éstas fueron indicaciones absolutas, pero hoy en día con los avances en la fijación interna, el uso de materiales intrasinusales para soporte de piso orbitario y fracturas cigomáticas inestables es más reducido. Un número de materiales ha sido colocado como fijación interna para las fracturas cigomáticas, así como balones, dren de Penrose, tubos plásticos y tiras de gasa; ha existido un gran debate sobre la efectividad de estos materiales dentro del seno maxilar en la corrección de las fracturas del complejo cigomático. El uso del balón insertado a través de un abordaje transnasal ha reportado resultados variados..$^{25}$
Wangerin y colaboradores usaron una sonda de Foley y encontraron que esta técnica era inefectiva porque al reducir las fracturas éstas se mantenían inestables. ${ }^{26}$ Fain y colaboradores, además de usar la sonda de Foley, usaron alambres y placas en la sutura fronto-cigomática y obtuvieron buenos resultados. ${ }^{27}$ Por otra parte, McCoy y colaboradores reportaron un caso en el cual al empacar en el seno maxilar una sonda de Foley desplazaron segmentos óseos y lesionaron el nervio óptico causando ceguera, por eso consideraron este método como antiguo, peligroso e ineficaz. ${ }^{28}$ Finalmente, Finlay y colaboradores publicaron que pacientes tratados con empaques de gasa en el seno maxilar produjeron mayores disestesias del nervio infraorbitario e infecciones sinusales comparados con pacientes que fueron fijados con pines externos. ${ }^{29}$

A pesar de su utilidad, la sonda de Foley puede tener complicaciones secundarias al procedimiento quirúrgico como diplopías persistentes, fístulas antrales, olor fétido, comprensión del nervio óptico por los fragmentos óseos y sinusitis crónica. ${ }^{17}$

De acuerdo con lo citado se puede concluir que el uso de la sonda de Foley es útil en la reducción y estabilización de las fracturas órbito-malares, logra la elevación del globo ocular cuando se presenta un desplazamiento del contenido periorbitario en el seno maxilar. Sin embargo, algunos autores la consideran contraindicada por sus posibles efectos adversos y porque requiere la habilidad del cirujano.

\section{BIBLIOGRAFÍA}

1. Castillo D, Mogensen M, Blanco S, Kauan M, Gudiño R. Prevalencia de fracturas en los maxilares de los pacientes que acudieron al servicio de cirugía maxilofacial del Hospital Clínico Universitario (HCU). Acta Odontol Venez 2006; 44: 357 - 363.

2. Olate S, Alister HJP, Chaves Netto DD, Jaimez M, Mazzonetto R. Reconstruccion de piso de órbita con malla de titanio en trauma de tercio medio facial. Int J Odontostomat 2008; 2: 163 - 170.

3. Yupanqui A, Martínez AY. Injerto autólogo de calota craneana para reconstrucción de piso de órbita en trauma facial. Reporte de caso. Rev Estomatol Herediana 2005; 15: $168-170$.

4. Avello F, Avello A. Nueva clasificación de las fracturas de trazo unilateral. An Fac Med Lima 2007; 68: 75 - 79.

5. Vila N, Sanz J, Robredo J, Gil M, Atalaya F. Cirugía maxilofacial. Med Clin (Barc) 2005; 124: 26 - 28.

6. Casarramona F, Belda V, Cruz J, Pou R, Cruz J, Daza M, Cuadrada C, Enfisema palpebral por fractura de la lámina papirácea del etmoides. Emergencias 2003;15:247-249

7. Caminal J, Prat J, Arruga J. Fracturas puras del suelo orbitario: actitud terapéutica. Publicaciones Nexus [en línea] 1994; [fecha de acceso: noviembre de 2009]. URL disponible en: http://www.nexusediciones.com/np_ ao_1994_4_3_002.htm 
8. Salej S, Pedraza B, Pinzón M. Manejo quirúrgico de las fracturas orbitarias. Hospital de San José, 1997-2002. Repertorio de Medicina y Cirugía 2003; 12:61 - 65

9. Ugrin C, Weil D. Transtornos oculomotores en las fracturas de órbita. [En línea] URL disponible en:http://www. plasticaocular.com/trabajos/órbita/trastornos_oculomotores_en_las_fracturas_orbitales

10. Burm J, Cheng $\mathrm{CH}$. Pure orbital blowout fractures: new concepts and importance of medial orbital blow out fracture. Plastic Reconstr Surg 1999; 103: 1839 - 1849.

11. Moore K, Dalley A. Anatomía con Orientación Clínica. Editorial Panamericana, 5ta. Edición; México. p. 960 - 973.

12. Tortora G, Derrickson B. Anatomía y Fisiología. Editorial Oxford, 9na. Edición, pág. 321 - 322.

13. Latarjet M, Ruiz A. Anatomía Humana. Editorial panamericana, 4ta. Edición, pag. 414-417.

14. Zambrano J, Leyva C. Lesiones oculares y perioculares asociadas a los traumatismos de órbita. Cir Plast Iberolatinoame 2007; 33: 233 - 242.

15. Rhee J, Kilde J, Yoganadan N, Pintar F. Orbital Blowout fractures experimental evidence for the pure hydraulic theory. Arch Facial Plast Surg 2002; 4: 98 - 101.

16. Muñoz J, Gutiérrez J, Zubizarreta F. Organización en el tratamiento del traumatismo panfacial y de las fracturas complejas del tercio medio. Cir Plast Iberolatinoam 2009; 35: $43-54$.

17. Díaz JM, Góngora R. Estudio comparativo sobre la estabilizacion de las fracturas cigomáticas. Rev Cubana Estomatol 1996; 33 [en línea]. [fecha de acceso: octubre de 2009]. URL disponible en: http://bvs.sld.cu/revistas/est/ vol33 3 96/est06396.htm

18. Simsek T, Keskin M, Eroglu L. The extended uses of Foley catheter in plastic surgery. Indian J Plast Surg 2006; 39: $111-116$.
19. Sveinsson E. Pure "blow-out" fractures of the orbital floor. J Laryngol Otol 1973; 87: 465 - 473.

20. Hong R, Yeon J, Shin S, Choi Y. Endoscopic versus external repair of orbital blowout fractures. Otolaryngology Head Neck Surg 2007; 136: 38 - 44.

21. Booth $\mathrm{W}$, Schendel P, Hausamen A. Maxillofacial surgery 1999; volume 1, pag 256 - 260, Churchill livingstone.

22. Rothman M, Simon E, Zoarski G, Zagardo M. Superior blowout fracture of the orbit: the blowup fracture. Am J Neuroradiol 1998; 19: 1448 - 1449.

23. Livingston $\mathrm{R}$, White $\mathrm{N}$, Catone $\mathrm{G}$, Thomas RF. Treatment of orbital fractures by an infraorbital-transantral approach. J Oral Surg 1975; 33: 586 - 590.

24. Kazanjian V, Converse J, Fracture of the zigoma. suspension method 1933; Surgical Treatment of Facial Injuries; Baltimore, 1974. pp 345 - 357

25. Rowe N, Williams J. Fractures of the zygomatic complex and orbit, Maxillofacial Injuries. Vol 1. London, ChurchillLivingstone, 1985, pp 435 - 537.

26. Wangerin $\mathrm{K}$, Busch $\mathrm{H}$, Conrad $\mathrm{H}$. Complication incidence of zigomatic bone fractures using different operation methods, oral and maxillofacial surgery: proceedings from the 8th international conference on oral and maxillofacil surgery, Chicago, Quinse 1985, pp 1133 - 1159

27. Fain J, Peri G, Thenoven V. The use of single frontozigomatic osteosynthesis plate and a sinus balloon in the repair of the lateral middle third of the face. J Maxillofac Surg 1981; 9: 188 - 193.

28. McCoy F, Chandler R, Magnan C. Fracture of the zigoma, Plast Reconstr Surg; 29: 381 - 398.

29. Finlay P, Ward R, Moos K. Morbidity association with the use of antral packs and external pins in the treatment of the unstable fracture of the zigomatic complex. Br J Oral Maxillofac Surg 1998; 22: $18-26$.

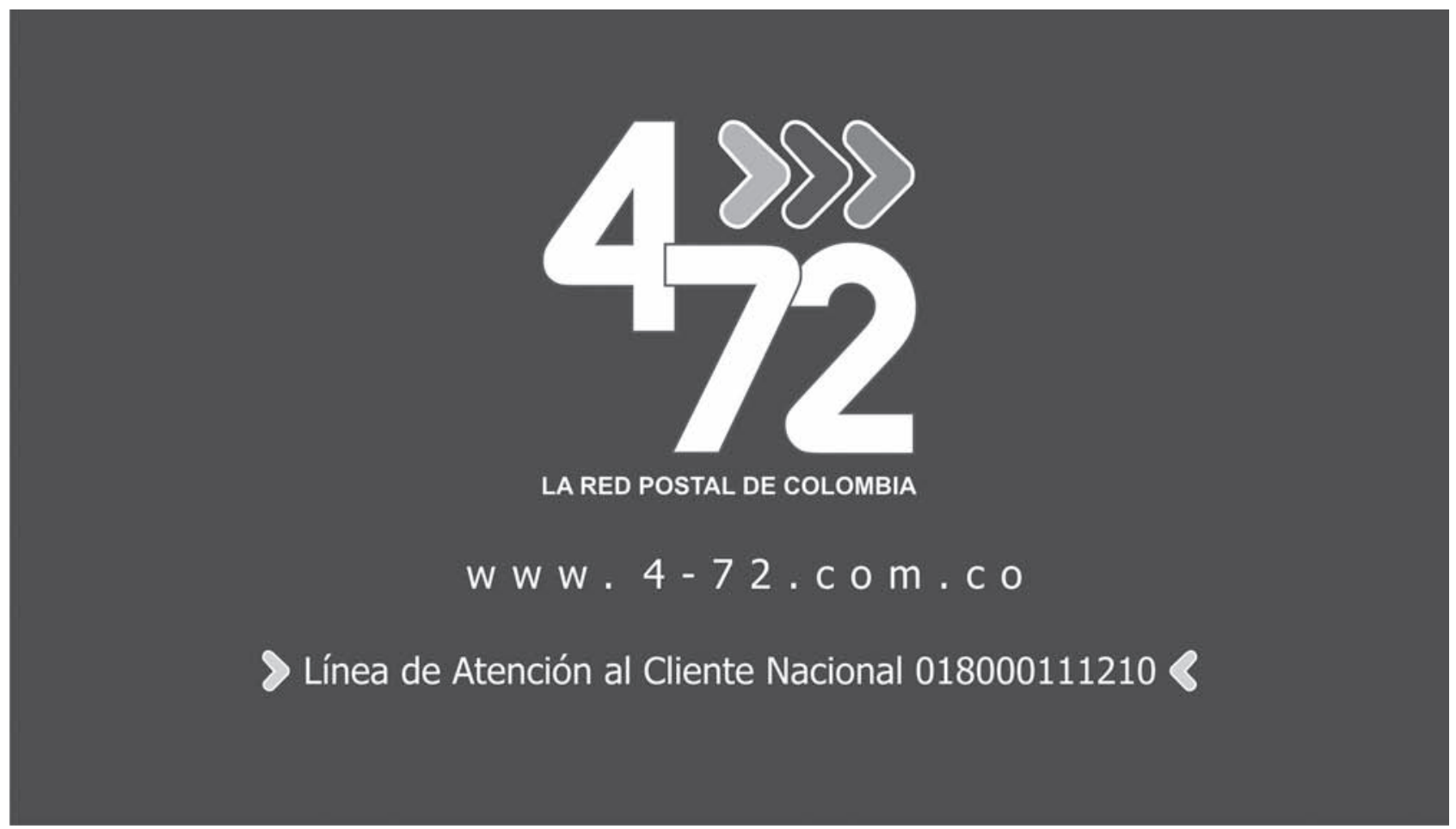

